\title{
An Aptamer-Based Antagonist against the Receptor for Advanced Glycation End-Products (RAGE) Blocks Development of Colorectal Cancer
}

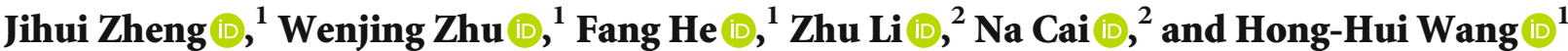 \\ ${ }^{1}$ College of Biology, Hunan University, Changsha, China \\ ${ }^{2}$ CellWay Bio, Changsha, China
}

Correspondence should be addressed to Na Cai; cainaya@hotmail.com and Hong-Hui Wang; wanghonghui@hnu.edu.cn

Received 3 March 2021; Accepted 23 April 2021; Published 5 May 2021

Academic Editor: Shuai Chen

Copyright (c) 2021 Jihui Zheng et al. This is an open access article distributed under the Creative Commons Attribution License, which permits unrestricted use, distribution, and reproduction in any medium, provided the original work is properly cited.

\begin{abstract}
Tumor angiogenesis plays a crucial role in colorectal cancer development. Dysregulation of the receptor for the advanced glycation end-products (RAGE) transmembrane signaling mediates inflammation, resulting in various cancers. However, the mechanism of the RAGE signaling pathway in modulating development of colorectal cancer has not been explored. In this study, an aptamer-based RAGE antagonist (Apt-RAGE) was used to inhibit interaction between RAGE and S100B, thus blocking downstream NFKB-mediated signal transduction. In vitro results showed that Apt-RAGE effectively inhibited S100B-dependent and S100B-independent RAGE/NF $\kappa$ B activation in colorectal HCT116 cancer cells, thus decreasing proliferation and migration of cells. Notably, expression and secretion of VEGF-A were inhibited, implying that Apt-RAGE can be used as an antiangiogenesis agent in tumor therapy. Moreover, Apt-RAGE inhibited tumor growth and microvasculature formation in colorectal tumor-bearing mice. Inhibition of angiogenesis by Apt-RAGE was positively correlated with suppression of the RAGE/NF $\kappa$ B/VEGF-A signaling. The findings of this study show that Apt-RAGE antagonist is a potential therapeutic agent for treatment of colorectal cancer.
\end{abstract}

\section{Introduction}

Colorectal cancer is a multifactorial disease that affects over four million people worldwide [1]. Previous studies report that the development of colorectal cancer is highly correlated with tumor angiogenesis [2] which is implicated in the pathological process of many critical diseases [3]. Inhibition of angiogenesis is associated with alleviation of cancer progression and metastasis $[4,5]$. New blood vessels formed during angiogenesis may contribute to inflammation-associated carcinogenesis, inducing tumor progression and tumor metastasis $[6,7]$. Unregulated release of different inflammatory mediators in the tumor microenvironment (TME) activates growth, proliferation, and migration of colorectal cancer cells [8]. In addition, inflammation mediators in TME induce abnormal angiogenesis by increasing secretion of vascular endothelial growth factor (VEGF). Moreover, the receptor for advanced glycation end-products (RAGE) is positively correlated with increased vessel density and progression of colorectal cancer, implying that the RAGE signaling pathway participates in VEGF-mediated angiogenesis [9].

RAGE is a type I transmembrane receptor present in diverse cell types and functions as a pattern-recognition receptor [10]. Interaction of RAGE with its various ligands (including AGE, HMGB1, and S100B) promotes cancer cell growth, invasion, and angiogenesis [3]. The S100 protein ligand activates the RAGE signaling thus promoting survival and proliferation of cancer cells [11]. In addition, overexpression of RAGE promotes colon cancer malignancy by accelerating proliferation and migration of cancer cells through activation of nuclear factor-kappa B- (NFkB-) mediated transcription leading to inflammation-associated carcinogenesis [12-14]. Furthermore, S100B plays an essential role in colon carcinogenesis by promoting $\mathrm{NF} \kappa \mathrm{B}$-mediated transcription through the RAGE signaling, affecting various phenotypes 
of cancer such as proliferation, metastasis, and angiogenesis [15]. Therefore, studies should explore novel specific therapeutic agents targeting $\mathrm{S} 100 \mathrm{~B} / \mathrm{RAGE} / \mathrm{NF} \kappa \mathrm{B}$ axis to block development of colorectal cancer.

Cell surface receptor targeting strategies have explored aptamers as novel candidates for targeted cancer therapy. Aptamers have various advantages over protein-based drugs, such as antibodies or peptides [16]. Aptamers are short and single-stranded oligonucleotides and have been reported to be antagonists against various target proteins through phylogenetic and exponential enrichment (SELEX) analysis [17]. Aptamers are chemical antibodies with three-dimensional structures; therefore, they have excellent thermal stability and high affinity and specificity for the homologous protein targets and display good biocompatibility $[18,19]$. Therefore, in this study, we developed an aptamer-based strategy for use as a molecular inhibitor of RAGE/NF $\kappa$ B/VEGF-A axis for suppression of inflammatory-induced angiogenesis associated with colorectal cancer progression.

The aim of this study was to design an aptamer against RAGE as a new antagonist to selectively inhibit RAGE/NF $\kappa \mathrm{B}$ signaling transductions. Analysis showed that the aptamerbased antagonist inhibited proliferation and migration of colorectal cancer cells induced by S100B. In addition, it decreased synthesis and secretion of VEGF-A protein, which is implicated in tumor angiogenesis. The in vivo results indicated that the aptamer of RAGE exhibited excellent inhibition activity on development of colorectal tumors, by suppressing angiogenesis and microvasculature formation in xenograft nude mice. The findings of this study, therefore, show that the novel aptamer against RAGE is a potential therapeutic agent for treatment of colorectal cancer.

\section{Material and Methods}

2.1. Reagents. The fetal bovine serum (FBS) was purchased from Biological Co., Ltd.; penicillin-streptomycin (100x), $0.25 \%$ trypsin-EDTA (1x), serum-free cryopreservation fluid, 3-(4,5-dimethyl-2-thiazolyl)-2,5-diphenyl-2-H-tetrazolium bromide (CCK-8), ECL luminescent agents, color pre-dyed protein marker, and antibody diluent were purchased from New Cell \& Molecular Biotech Co., Ltd.; recombinant protein S100B (E. coli, N-6His) was purchased from Novoprotein Scientific Inc. RIPA lysis buffer was purchased from Beyotime Biotechnology. Phosphatase inhibitors and protease inhibitors were from Bimake; nitrocellulose membrane was purchased from Merck Millipore Company; antibodies against phosphorylated NF $\kappa$ B p65 (Ser 536), phosphorylated Akt (Ser 473), and phosphorylated ERK1/2 (Thr202/Tyr204) were purchased from Cell Signaling Technology (Beverly, Massachusetts, USA). The polyclonal antibodies for VEGFA, RAGE, and CD31 were from Santa Cruz Biotechnology (Santa Cruz). The primary monoclonal antibody against $\alpha$ tubulin was purchased from CellWay technology (CellWay Bio). The secondary antibodies including HRP-conjugated goat anti-mouse IgG (H\&L) and HRP-conjugated goat antirabbit IgG (H\&L) were from Invitrogen. PrimeScript ${ }^{\mathrm{TM}} \mathrm{RT}$ reagent kit (perfect real time), Takara RR037A kit, EasyGeno rapid recombinant clone kit, endotoxin-free plasmid extrac- tion kit, and DNA gel recovery kit were obtained from Tiangen.

2.2. Cell Culture. HCT116 cell, a colon cancer cell line, was cultured in Dulbecco's modified Eagle's medium (DMEM) with $10 \%$ FBS and $1 \%$ penicillin/streptomycin. All cells were incubated in a humidified atmosphere at $37^{\circ} \mathrm{C}$ with $5 \% \mathrm{CO}_{2}$.

2.3. Preparation of Apt-RAGE. The aptamer against RAGE (Apt-RAGE) was adopted based on the systematic evolution of ligands by exponential enrichment (SELEX) previously. [20] Sequences of Apt-RAGE and the control DNA aptamer (Ctrl-aptamer) are as follows. Apt-RAGE: $5^{\prime}$-CCTGATATG GTGTCACCGCCGCCTTAGTATTGGTGTCTAC-3' and Ctrl-aptamer: $\quad 5^{\prime}$-TTCGGCCTGGGGGCGGCCAGTTCG GGTCCAGTCGCGGGAG-3'.

2.4. Molecular Cloning. The cloning primers (Supplemental Table 1) were designed according to the CDS sequence of the gene AGER (Homo sapiens gene of rage). The cDNA fragment of the RAGE was amplified by PCR and cloned into the plasmid of pcDNA 3.1 vector. The plasmids of pcDNA 3.1 RAGE were isolated and identified by Sanger sequencing (Tsingke Biotech Co., Ltd.).

2.5. Plasmid Transfection. Cells were inoculated and transfected into a 6-well plate culture dish (Wuxi NEST Biotechnology Co., Ltd.) with about $2 \times 10^{5}$ cells per well on the day before transfection, and the cell density could reach about $70-80 \%$ on the next day. The mixture of Lipo8000 ${ }^{\mathrm{TM}}$ Transfection reagent and plasmids was dripped into the cells in the six-well plate. After 6 hours, the culture medium was changed for $48 \mathrm{~h}$ culturing.

2.6. Western Blotting. Cells were seeded in a 6-well plate culture dish (NEST Biotech Co., Ltd.). Before treatment, the cells were starved for $24 \mathrm{~h}$ by incubation with the DMEM containing $0.2 \%$ FBS. Subsequently, the cells were pretreated with Ctrl-aptamer or Apt-RAGE for one hour, then treated with $\mathrm{S} 100 \mathrm{~B}$ for $30 \mathrm{~min}$ in the incubator, and then lysed with RIPA lysis buffer. The cell lysates were centrifuged at $14000 \mathrm{rcf}$ for $10 \mathrm{~min}$ at $4^{\circ} \mathrm{C}$ and separated by $10 \%$ SDSPAGE electrophoresis followed by transferring to a nitrocellulose membrane by semidry electrophoretic transfer unit. Each membrane was blocked with 5\% skim milk in PBST (1× PBS with $0.1 \%$ Tween-20) at room temperature for $1.5 \mathrm{~h}$ and incubated with different primary antibodies (1:1000 dilution) overnight at $4^{\circ} \mathrm{C}$. Subsequently, the membranes were incubated with horseradish peroxidaseconjugated secondary antibodies at room temperature for $1 \mathrm{~h}$. Finally, the membranes were reacted with ECL substrate solution (NCM Biotech Co., Ltd.) and the chemiluminescent images were acquired and analyzed using Bio-Imaging Systems (MicroChemi4.2).

2.7. Cell Viability Assay. The CCK8 assay was carried out to determine relative cell viability. [21] HCT116 cells were seeded at $5 \times 10^{3}$ cells/well on a 96-well plate culture dish (Wuxi NEST Biotechnology Co., Ltd.) and allowed to adhere for $24 \mathrm{~h}$ at $37^{\circ} \mathrm{C}$ in $5 \% \mathrm{CO}_{2}$. The cells were pretreated with 


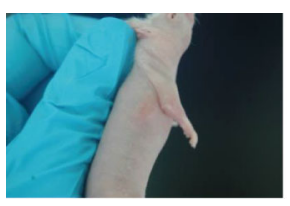

0 Day

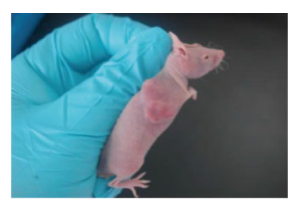

12 Days

(a)

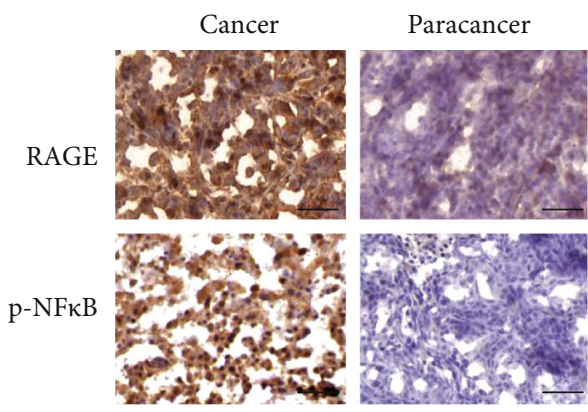

(b)

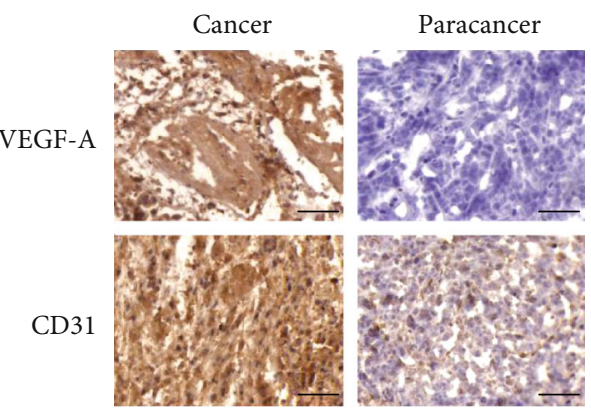

(c)

FIGURE 1: Association of RAGE expression with tumor angiogenesis in colorectal cancer development. (a) HCT116 cells were intradermally injected into the upper flank of 6-week female nude mice to establish colorectal cancer xenograft model. (b) Immunohistochemical analysis of cancer tissues and the paracancer tissues from nude mice using antibodies against RAGE and p-NF $\kappa$ B. Scale bar: $50 \mu$ m. (c) Immunohistochemical analysis of cancer tissues and the paracancer tissues from nude mice using antibodies against VEGF-A and CD31. Scale bar: $50 \mu \mathrm{m}$.

Apt-RAGE $(100 \mathrm{nM})$ and then treated with S100B ( $2 \mathrm{ugs} / \mathrm{mL}$ ), S100B within the fresh medium for $24 \mathrm{~h}, 48 \mathrm{~h}$, or $72 \mathrm{~h}$. After the incubation, cells within the 96-well plate were incubated with CCK8 solution for $1-3 \mathrm{~h}$ at $37^{\circ} \mathrm{C}$ in $5 \%$ $\mathrm{CO}_{2}$, followed by measurement using a GENios Microplate Reader (TECAN) at the absorbance at $450 \mathrm{~nm}$.

2.8. Wound Healing Assay. $3 \times 10^{4}$ HCT116 cells were seeded into a 12 -well plate culture dish (Wuxi NEST Biotechnology Co., Ltd.) for $90 \%$ confluence and scratched using a $200 \mu \mathrm{L}$ tip. After scratching, the cells were washed with PBS twice, and then the medium containing Apt-RAGE (100 nM) was added. After $1 \mathrm{~h}, \mathrm{~S} 100 \mathrm{~B}$ was added to the culture medium. Under an inverted microscope, the scratched cells were photographed after $0 \mathrm{~h}, 24 \mathrm{~h}$, and $48 \mathrm{~h}$. The wound closure rate was calculated as follows: Migration rate $(\%)=($ Scratch distance at $0 \mathrm{~h}-\mathrm{Scratch}$ distance at indicated time)/Scratch distance at $0 \mathrm{~h} \times 100$.

2.9. Transwell Assay. The migration ability of HCT116 cells was assessed by using Transwell chamber (BD Biosciences, San Diego, CA). $500 \mu \mathrm{L}$ of complete medium containing $10 \%$ FBS was supplemented into the lower chamber (the bottom of a 24-well plate), then the medium containing Apt-RAGE $(100 \mathrm{nM})$ or Ctrl-aptamer. Next, $5 \times 10^{4}$ cells/ well were added into the upper chamber. S100B was added into the lower chamber. After $24 \mathrm{~h}$ incubation, the migrated cells were stained with crystal violet. Five random fields at 200x magnification were used for cell counting for each membrane.

2.10. Measurement of VEGF-A by ELISA. ELISA was performed to determine VEGF-A production according to the user's manual of a Human VEGF-A ELISA kit (ABclonal Technology).

2.11. Xenograft Studies. The animal experiments were approved by the ethical committee of College of Biology, Hunan University, China, and performed according to the Guide for the Care and Use of Laboratory Animals of the National Institutes of Health. The HCT116 cells $\left(2 \times 10^{6}\right.$ cells) were intradermally injected into the upper flank of female 6-week nude mice $(n=20)$. 2 days posttumor inoculation, Apt-RAGE (38.4 pmol/day/g body weight, $n=5$ ) or vehicle $(n=5)$ was injected adjacent to the tumor daily for 12 days. The volume of tumors and body weight were measured daily. The tumor volume $\left(\mathrm{mm}^{3}\right)=[($ width $) / 2 \times$ length/2] $\mathrm{mm}^{3}$. At 12 days posttumor inoculation, mice were humanely sacrificed by isoflurane inhalation, and the HCT116 tumor section was excised for immunohistochemical staining.

2.12. Immunohistochemical Staining. Harvested tumors and paracancerous tissue were embedded in the optimal cutting temperature compound (OCT, Tissue-Tek, Sakura), stored at $-80^{\circ} \mathrm{C}$. Immunohistochemistry was carried out using a two-step ELISA Kit (mouse/rabbit-enhanced polymer system) (ZSGB-BIO). Primary antibodies include RAGE (1:50 dilution), VEGF-A (1:50 dilution), $\mathrm{p}-\mathrm{NF} \kappa \mathrm{B}$ (1:50 dilution), and CD31 (1:50 dilution). The specimens were stained using the $\mathrm{DAB}$ kit (ZSGB-BIO) and then imaged using a digital slicing system (Pannoramic MIDI).

2.13. Statistical Analysis. All values were presented as the means \pm SEM. Statistical significance was evaluated using the Student $t$-test for paired comparison (GraphPad Prism $6) ;{ }^{*} p<0.05$ and ${ }^{* *} p<0.01$ were considered to be significant. 


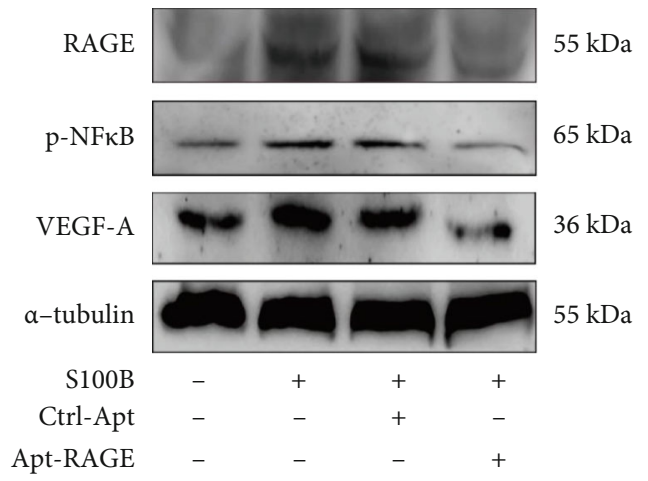

(a)

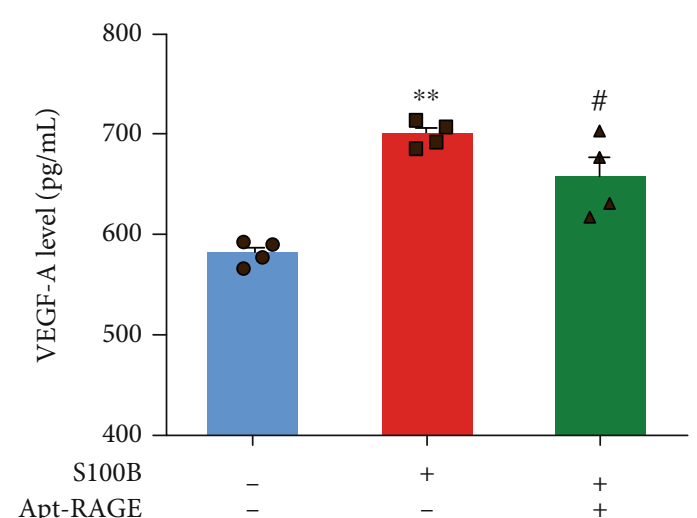

(b)

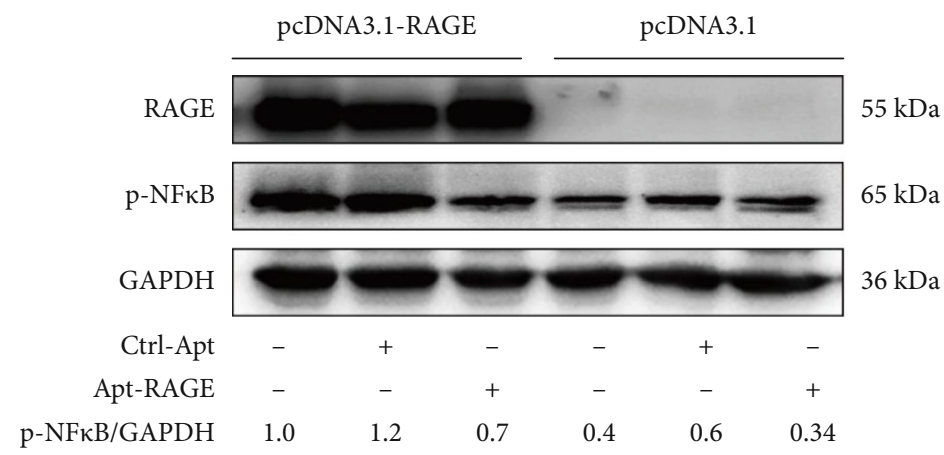

(c)

FIGURE 2: Inhibitory effect of Apt-RAGE on S100B-induced synthesis and secretion of VEGF-A protein. (a) HCT116 cells were starved and pretreated with $100 \mathrm{nM}$ Apt-RAGE or Ctrl-Apt for $90 \mathrm{~min}$ in the presence of S100B $(2 \mu \mathrm{g} / \mathrm{mL})$. Phosphorylation of NF $\kappa \mathrm{B}$, VEGF-A, and RAGE was examined using western blotting. (b) ELISA assay was used to determine VEGF-A release in the culture medium treated with S100B $(2 \mu \mathrm{g} / \mathrm{mL})$ in the presence or absence of Apt-RAGE $(100 \mathrm{nM})$ or Ctrl-Apt $(100 \mathrm{nM})$. Data are presented as the means \pm SEM, ${ }^{* *} p<$ 0.01 vs. untreated control and ${ }^{*} p<0.05$ in Apt-RAGE vs. S100B. (c) Apt-RAGE inhibited S100B-independent phosphorylation of NF $\kappa B$ in RAGE-overexpressed cells. HCT116 cells transfected with pcDNA3.1-RAGE or pcDNA3.1 for $48 \mathrm{~h}$ and incubation with Apt-RAGE $(100 \mathrm{nM})$ for $20 \mathrm{~min}$. Phosphorylation of $\mathrm{NF} \kappa \mathrm{B}$ and the expression of VEGF-A and RAGE were examined using western blotting.

\section{Results}

3.1. RAGE Expression Correlates with Microvasculature Formation in Colorectal Cancer. Tumor-associated angiogenesis is associated with tumor growth and development in vivo [22]. A colorectal tumor-bearing nude mouse model was established to explore the role of RAGE in tumorassociated angiogenesis (Figure 1(a)). Expression of RAGE and phosphorylation of NF $\kappa$ B were analyzed through immunohistochemistry staining of tumor specimens prepared post 12 days after tumor inoculation days. Analysis of staining results showed increase in expression level of RAGE protein and significant phosphorylation of $\mathrm{NF} \kappa \mathrm{B}$ in colorectal tumor tissue compared with those in paracancer normal tissue (Figure 1(b)). Notably, CD31-positive blood vessels formed adjacent to tumor tissues, and a significant increase in the level of VEGF-A was observed compared with those in the adjacent normal tissue (Figure 1(c)). These findings imply that development of colorectal cancer may be mediated by $\mathrm{RAGE} / \mathrm{NF} \kappa \mathrm{B} / \mathrm{VEGF}-\mathrm{A}$ axis, which plays a role in promoting tumor angiogenesis.
3.2. Apt-RAGE Blocks the NFkB Signaling Pathway and VEGF-A Secretion. The role of RAGE signaling in tumorigenesis was explored using in vitro cultured colorectal cells. S100B, a ligand of RAGE and a known mediator of inflammation, significantly induced phosphorylation of $\mathrm{NF} \kappa \mathrm{B}$ and expression of VEGF-A (Figure 2(a)). It has been previously reported that the $\mathrm{RAGE}-\mathrm{NF} \kappa \mathrm{B}$ signaling plays an essential role in VEGF-A secretion [23]. In this study, we explored the relationship between S100B and VEGF-A secretion using HCT116 cells. ELISA analysis showed that S100B enhanced VEGF-A secretion in the culture medium (Figure 2(d)), implying that VEGF-A secretion is mediated by induction of the RAGE/NF $\kappa$ B signaling pathway by $\mathrm{S} 100 \mathrm{~B}$. We proposed that the aptamer-based RAGE antagonist (Apt-RAGE) may inhibit the interaction between RAGE and S100B to block downstream NF $\kappa$ B-mediated signal transduction. The aptamer against RAGE was previously screened and was used to block the AGE-RAGE signaling, which efficiently attenuated the development experimental diabetic nephropathy [20]. We characterized the stability of Apt-RAGE in $10 \%$ serum and found that the Apt-RAGE remained stable 


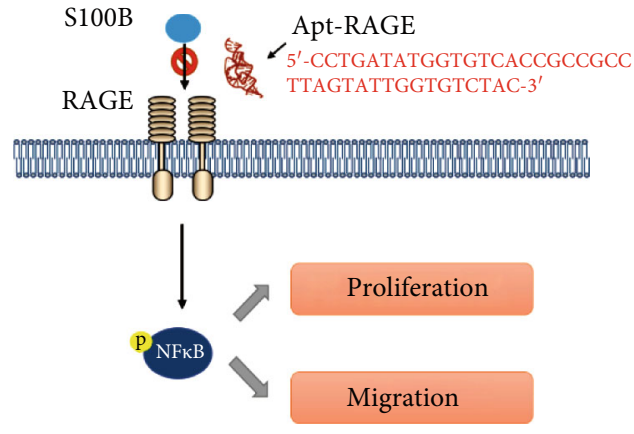

(a)

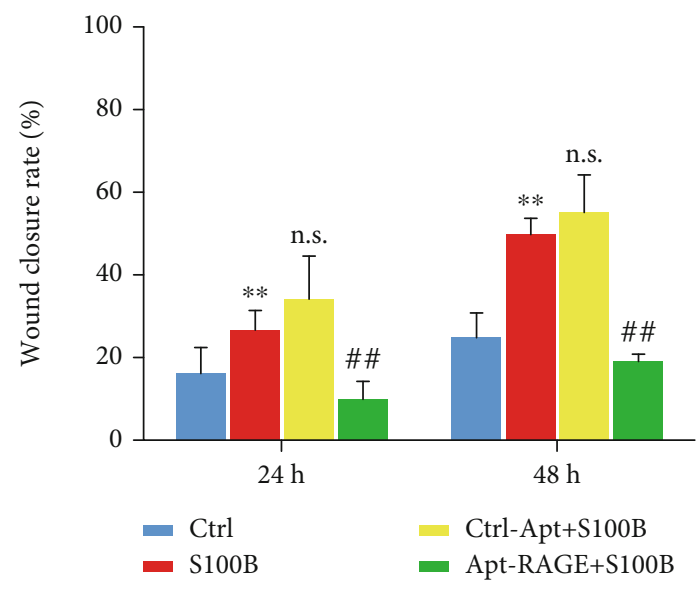

(c)

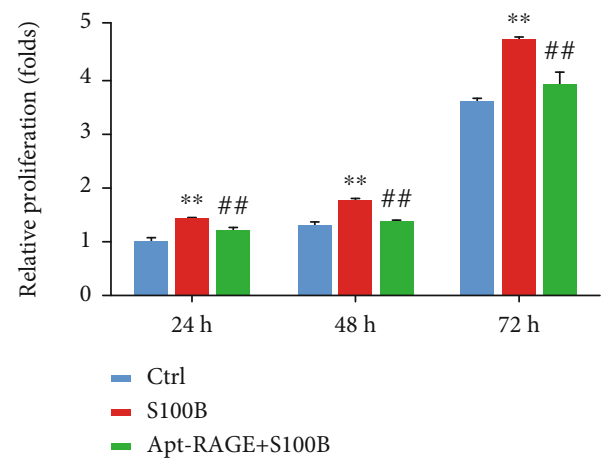

(b)
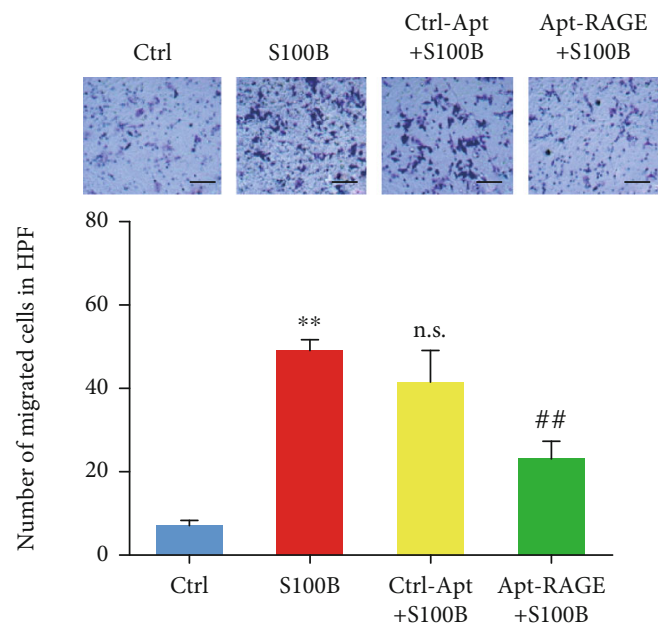

(d)

FIGURE 3: Apt-RAGE inhibited proliferation and migration of colorectal cancer cells. (a) Schematic illustration of Apt-RAGE effect on proliferation and migration of cancer cells. (b) Effect of Apt-RAGE (100 nM) on proliferation of HCT116 cells promoted by S100B $(2 \mu \mathrm{g} / \mathrm{mL})$ was detected by CCK8 cell counting kit at $24 \mathrm{~h}, 48 \mathrm{~h}$, and $72 \mathrm{~h}$. Data are presented as the means $\pm \mathrm{SEM}$, ${ }^{*} p<0.01 \mathrm{vs}$. untreated control and ${ }^{\#} p<0.05$ vs. S100B. (c) Quantitation of the effect of Apt-RAGE (100 nM) on migration induced by S100B $(2 \mu \mathrm{g} / \mathrm{mL})$ in wound healing assay at $24 \mathrm{~h}$ or $48 \mathrm{~h} .{ }^{*} p<0.01$ vs. untreated group and ${ }^{\# \#} p<0.01$ vs. S100B-treated group. n.s. indicates that the difference is not significant compared with the S100B-treated group. (d) Quantitative analysis of the effect of Apt-RAGE (100 nM) on directional migration induced by S100B $(2 \mu \mathrm{g} / \mathrm{mL})$ evaluated using Transwell assay. Upper panel: representative image of the membrane with migrated cells, scale bar: $200 \mu \mathrm{m}$. Lower panel: analysis of migrated cells using Transwell assay. Data are presented as the means $\pm \mathrm{SEM},{ }^{* *} p<0.01 \mathrm{vs}$. untreated control, ${ }^{\# \#} p<0.01$ vs. S100B-treated group. n.s. indicates that the difference is not significant compared with the S100Btreated group.

without degradation in 5 hours, which could be enough for cell experiment and in vivo study (Figure S1). Based on the Mfold software simulation, two predicted secondary structures were generated by free energy minimization using the RNA folding algorithm (Figure S2). The calculated free energy for each aptamer is $\Delta \mathrm{GA}=-2.07 \mathrm{kcal} / \mathrm{mol}$ and $\Delta \mathrm{GB}=-1.39 \mathrm{kcal} / \mathrm{mol}$. Notably, pretreatment of HCT116 cells with $100 \mathrm{nM}$ Apt-RAGE significantly inhibited S100B-induced phosphorylation of NF $\kappa$ B and increased in VEGF-A protein level compared with the Ctrl-Apt-treated group (Figure 2(a)). We also examined other signaling pathways and found that Apt-RAGE failed to affect the Akt and ERK signaling, suggesting the specificity of the Apt-RAGE to block the RAGE/NF $\kappa$ B signaling (Figure S3). Furthermore, we confirmed that Apt-RAGE suppressed secretion of VEGF-A in culture medium (Figure 2(b)). These findings imply that Apt-RAGE inhibits VEGF-A release by inhibiting the RAGE/NF $\kappa$ B signaling pathway.

A previous study reports that overexpression of RAGE affects downstream signaling thus promoting proliferation of hepatic cancer cells in a ligand-independent manner [24]. To explore the effect of high overexpression of RAGE on the NF $\kappa \mathrm{B}$ signaling in colorectal cancer cells, HCT116 cells were transfected with pcDNA-3.1-RAGE to overexpress human RAGE protein. Overexpression of RAGE significantly increased phosphorylation of $\mathrm{NF} \kappa \mathrm{B}$ in pcDNA 3.1 RAGEtransfected cells compared with the control cells transfected with an empty vector (pcDNA 3.1) lacking RAGE (Figure 2(c)). Analysis showed that Apt-RAGE significantly inhibited phosphorylation of $\mathrm{NF} \kappa \mathrm{B}$ in HCT116 cells 


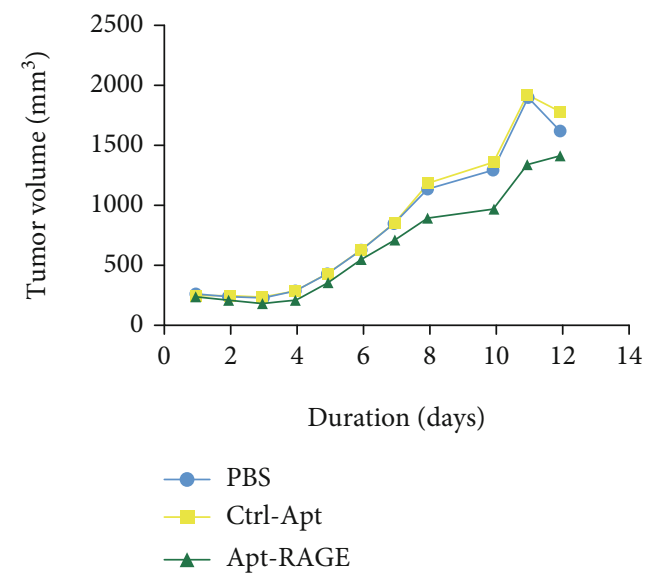

(a)

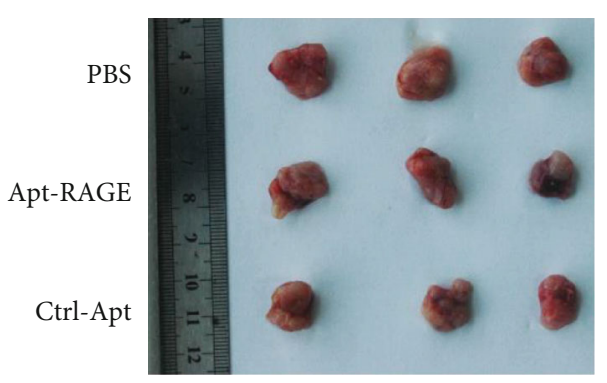

(b)

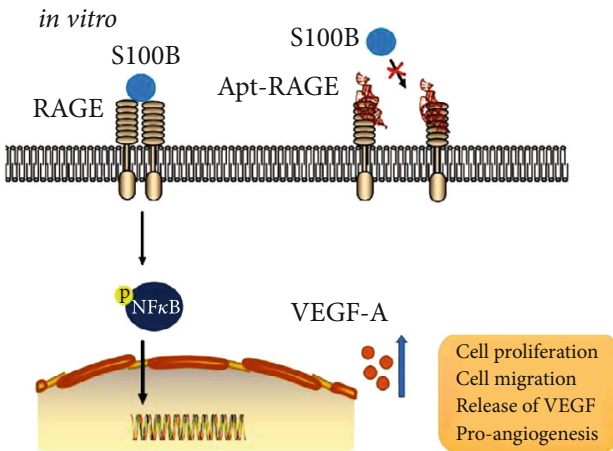

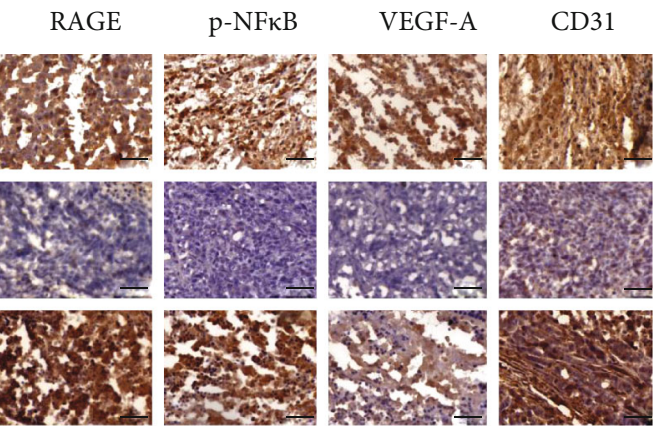

(c)

in vivo

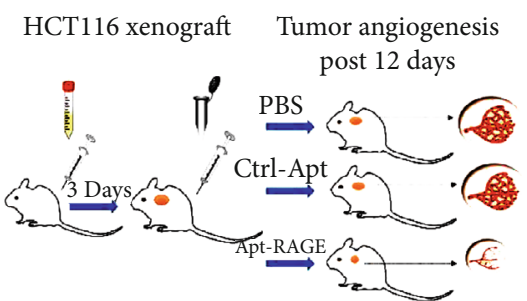

(d)

FIGURE 4: Effects of Apt-RAGE on angiogenesis of HCT116 cells xenograft nude mice. (a) The average volume of tumors treated with PBS $(n=5)$, Ctrl-Apt $(n=5)$, or Apt-RAGE $(n=5)$. Tumor volume was measured until the end of the experiments. (b) Images of representative tumors. (c) IHC staining was performed with RAGE, p-NFאB, VEGF, or CD31 on the frozen sections. Scale bar: $50 \mu \mathrm{m}$. (d) Schematic illustration of the role of Apt-RAGE to inhibit in vivo tumor angiogenesis (right) by blocking the $\mathrm{S} 100 \mathrm{~B} / \mathrm{RAGE} / \mathrm{NF} \kappa \mathrm{B} / \mathrm{VEGF}-\mathrm{A}$ signaling pathway (left).

with overexpressed RAGE (Figure 2(c)). This finding indicates that Apt-RAGE can be used as a potent antagonist for RAGE protein to inhibit the S100B-dependent and S100B-independent RAGE/NF $\kappa$ B signaling.

3.3. Apt-RAGE Inhibits S100B-Induced Proliferation and Migration. Further, we explored the role of S100B on proliferation and migration of colorectal cancer cells (Figure 3(a)). Analysis showed that S100B significantly increased proliferation of HCT116 cells $(2 \mu \mathrm{g} / \mathrm{mL})$ compared with that of controls (Figure 3(b)). Pretreatment with $100 \mathrm{nM}$ Apt-RAGE significantly reduced proliferation of HCT116 cells (Figure 3(b)). To explore the effect of S100B on cell migration, a wound scratch assay was performed on a $2 \mathrm{D}$ interface. Analysis showed a significant increase in cell migration rate after treatment with S100B in a time-dependent manner compared with control cells (Figure 3(c), Figure S4). However, pretreatment with Apt-RAGE (100 nM) significantly reduced S100B-induced wound closure of HCT116 cells. Further, Transwell assay was performed to investigate the effect of Apt-RAGE on S100B-induced cell migration using a 3D interface. Analysis showed that Apt-RAGE significantly 
decreased the number of migrated cells compared with that of the S100B-treated group (Figure 3(d)). These findings show that Apt-RAGE effectively inhibits S100B-induced proliferation and migration of colorectal cancer cells.

3.4. Apt-RAGE Retards Development of Colorectal Cancer by Modulating Angiogenesis In Vivo. To investigate the effects of Apt-RAGE aptamer as an antagonistic agent in vivo, a colorectal tumor xenograft model was constructed. In summary, HCT116 cells were injected subcutaneously in mice to induce tumors. The tumor-bearing mice were administered with Apt-RAGE or Ctrl-aptamer daily for 12 days. The tumor volume from the mice treated with the AptRAGE was significantly smaller compared with that of the control group (Figure 4(a)). In the first four days, the tumor growth in the experimental group was similar to the control group, whereas growth gradually decreased from day five, compared with the PBS group and the Ctrl-aptamer group (Figure 4(b)). Analysis showed that Apt-RAGE significantly inhibited tumor growth. Furthermore, immunohistochemical analysis was performed showing that Apt-RAGE inhibited the RAGE level and phosphorylation of $\mathrm{NF} \kappa \mathrm{B}$, resulting in low VEGF-A levels compared with levels in the control group (Figure 4(c)). These findings indicate that Apt-RAGE inhibits tumor angiogenesis by blocking $\mathrm{RAGE} / \mathrm{NF} \kappa \mathrm{B}$ signal transduction.

\section{Discussion}

Development of colorectal cancer is associated with aberrant RAGE activation through angiogenesis-promoting TME inflammation. Therefore, RAGE is a potential therapeutic target for colorectal cancer treatment $[9,15]$. RAGE binds to multiple ligands, including advanced glycation endproducts (AGEs) and S100 proteins. After binding these ligands, it activates the downstream $\mathrm{NF} \kappa \mathrm{B}$ pathway which is implicated in regulation of cell proliferation, survival, differentiation, and autophagy $[25,26]$. In the present study, RAGE expression level was determined and the association with microvessel density in colorectal cancer tissue specimens was explored. The findings of this study showed that colorectal cancer tissues express high RAGE protein levels, which are positively correlated with increased microvessel density. In addition, phosphorylation $\mathrm{NF} \kappa \mathrm{B}$ pattern was positively correlated with expression levels of VEGF and CD31 proteins in colorectal cancer tissue specimens.

$\mathrm{S} 100 \mathrm{~B}$ is used as a diagnostic marker for inflammatory malignant tumors. The S100B-induced signaling is positively correlated with development of various tumors [27, 28]. Moreover, VEGF-A promotes tumor angiogenesis during development of colorectal cancer [15]. This study explored the role of the S100B/RAGE signaling on cell viability, migration, and angiogenesis and progression of colorectal cancer. The findings of this study show that increased S100B protein levels are correlated with significant increase in proliferation and migration of human colon cancer cell in vitro. In addition, S100B activity significantly increased VEGF-A secretion from cultured cells. Apt-RAGE inhibited the RAGE signaling in colorectal cancer cells, thus inhibiting activation of $\mathrm{NF} \kappa \mathrm{B}$, tumor cell proliferation, migration, and release of VEGF-A. A previous study reported that RAGE is highly expressed in hepatoma cancer cells and is implicated in promoting proliferation of hepatoma cancer cells [24]. Similarly, the findings of this study showed that RAGE overexpression induces phosphorylation of $\mathrm{NF} \kappa \mathrm{B}$ in the absence of S100B. Notably, analysis showed that Apt-RAGE inhibits S100B-independent $\mathrm{NF} \kappa \mathrm{B}$ activation through inhibition of RAGE expression. Furthermore, the findings of this study indicate that Apt-RAGE inhibits S100B-RAGE-mediated angiogenesis by inhibiting S100B-induced activation of NF $\kappa$ B. The inhibitory effect of Apt-RAGE on in vitro tumor angiogenesis was confirmed in vivo (Figure 4). In vivo experiments showed that AptRAGE inhibits phosphorylation of $\mathrm{NF} \kappa \mathrm{B}$ and expression of VEGF, thus decreasing microvasculature which was analyzed through CD31-positive staining of the vascular endothelium in colorectal tumor specimens.

In conclusion, the findings of this study show that AptRAGE, an antagonist for RAGE, significantly inhibits synthesis and secretion of VEGF-A protein by inhibiting the NF $\kappa \mathrm{B}$ pathway in human colon cancer cells. Therefore, inhibition of Apt-RAGE on VEGF-A-mediated angiogenesis significantly decreases formation of microvasculature around tumors in xenograft model. In addition, Apt-RAGE inhibited S100B-dependent activation of proliferation and migration of colorectal cancer cells, which are critical events for cancer cells to adapt to the TME during tumor progression (Figure 4(d)). To the best of our knowledge, this is first study to report that Apt-RAGE inhibits proangiogenic and proliferative features of colorectal cancer cells. These results provide a basis for selective targeting of S100B/RAGE signaling using aptamer which is a novel approach to develop novel nucleic acid drugs for colon cancer therapy.

\section{Data Availability}

The quantification data used to support the findings of this study are included within the article.

\section{Conflicts of Interest}

The authors declare no competing financial interests.

\section{Acknowledgments}

This work was supported by the Natural Science Foundation of Hunan Province (Grant No. 2020JJ4383), the Innovation-Oriented Advanced Technology and Industrial Technology Program Project of Hunan Province (Grant No. 2020SK2017), and the Changsha Municipal Natural Science Foundation (Grant No. kq2014043).

\section{Supplementary Materials}

Supplemental Table 1: primer sequences for plasmid construction. Figure S1: the characterization of Apt-RAGE. (A) The molecular size of Apt-RAGE (Apt-RAGE) and Ctrl-aptamer (Ctrl-Apt). (B) The serum stability of AptRAGE. Figure S2: schematic representations of the secondary 
structures of the Apt-RAGE. Predicted secondary structures were generated by free energy minimization using the RNA folding algorithm Mfold (calculated free energy for each aptamer: $\Delta \mathrm{GA}=-2.07 \mathrm{kcal} / \mathrm{mol}$ and $\Delta \mathrm{GB}=-1.39 \mathrm{kcal} / \mathrm{mol}$. Figure S3: effect of Apt-RAGE on the AKT and ERK signaling pathway in cultured HCT116 cells. Figure S4: effect of Apt-RAGE $(100 \mathrm{nM})$ on the migration induced by S100B $(2 \mu \mathrm{g} / \mathrm{mL})$ was detected by wound healing assay at $24 \mathrm{~h}$ or 48 h. Scale bar: $50 \mu \mathrm{m}$. (Supplementary Materials)

\section{References}

[1] C. Lucas, N. Barnich, and H. Nguyen, "Microbiota, inflammation and colorectal cancer," International Journal of Molecular Sciences, vol. 18, no. 6, p. 1310, 2017.

[2] K. A. Rmali, M. C. A. Puntis, and W. G. Jiang, "Tumour-associated angiogenesis in human colorectal cancer," Colorectal Disease, vol. 9, no. 1, pp. 3-14, 2007.

[3] M. B. Majnooni, S. Fakhri, A. Smeriglio et al., "Antiangiogenic effects of coumarins against cancer: from chemistry to medicine," Molecules, vol. 24, no. 23, p. 4278, 2019.

[4] Z. Li, W. Yan-qing, Y. Xiao et al., "Exosomes secreted by chemoresistant ovarian cancer cells promote angiogenesis," $J$. Ovarian. Res., vol. 14, no. 1, p. 7, 2021.

[5] K. Ganesh and J. Massagué, "Targeting metastatic cancer," Nature Medicine, vol. 27, no. 1, pp. 34-44, 2021.

[6] L. Rodriguez-Caso, A. Reyes-Palomares, F. Sanchez-Jimenez, A. R. Quesada, and M. A. Medina, "What is known on angiogenesis-related rare diseases? A systematic review of literature," Journal of Cellular and Molecular Medicine, vol. 16, no. 12, pp. 2872-2893, 2012.

[7] K. Cole, K. Pravoverov, and J. E. Talmadge, "Role of myeloidderived suppressor cells in metastasis," Cancer and Metastasis Reviews, 2021.

[8] V. Catalano, A. Turdo, S. Di Franco, F. Dieli, M. Todaro, and G. Stassi, "Tumor and its microenvironment: a synergistic interplay," Seminars in Cancer Biology, vol. 23, no. 6, pp. 522-532, 2013.

[9] H. Liang, Y. Zhong, S. Zhou, and L. Peng, "Knockdown of RAGE expression inhibits colorectal cancer cell invasion and suppresses angiogenesis in vitro and in vivo," Cancer Letters, vol. 313, no. 1, pp. 91-98, 2011.

[10] P. Swami, S. Thiyagarajan, A. Vidger, V. S. K. Indurthi, S. W. Vetter, and E. Leclerc, "RAGE up-regulation differently affects cell proliferation and migration in pancreatic cancer cells," International Journal of Molecular Sciences, vol. 21, no. 20, p. 7723, 2020.

[11] M. T. T. Win, Y. Yamamoto, S. Munesue et al., "Regulation of RAGE for attenuating progression of diabetic vascular complications," Experimental Diabetes Research, vol. 2012, Article ID 894605, 8 pages, 2012.

[12] M. K. Fuentes, S. S. Nigavekar, T. Arumugam et al., "RAGE activation by $\mathrm{S} 100 \mathrm{P}$ in colon cancer stimulates growth, migration, and cell signaling pathways," Diseases of the Colon and Rectum, vol. 50, no. 8, pp. 1230-1240, 2007.

[13] C. Cirillo, G. Sarnelli, G. Esposito, F. Turco, L. Steardo, and R. Cuomo, "S100B protein in the gut: the evidence for enteroglial-sustained intestinal inflammation," World Journal of Gastroenterology, vol. 17, no. 10, pp. 1261-1266, 2011.

[14] P. Moravkova, D. Kohoutova, S. Rejchrt, J. Cyrany, and J. Bures, "Role of S100 proteins in colorectal carcinogenesis,"
Gastroenterology Research and Practice, vol. 2016, Article ID 2632703, 7 pages, 2016.

[15] L. Seguella, R. Capuano, M. Pesce et al., "S100B protein stimulates proliferation and angiogenic mediators release through RAGE/pAkt/mTOR pathway in human colon adenocarcinoma Caco-2 cells," International Journal of Molecular Sciences, vol. 20, no. 13, p. 3240, 2019.

[16] G. Zhu and X. Chen, "Aptamer-based targeted therapy," Advanced Drug Delivery Reviews, vol. 134, pp. 65-78, 2018.

[17] M. Song, G. Li, Q. Zhang, J. Liu, and Q. Huang, "De novo postSELEX optimization of a G-quadruplex DNA aptamer binding to marine toxin gonyautoxin 1/4," Computational and Structural Biotechnology Journal, vol. 18, pp. 3425-3433, 2020.

[18] H. Sun and Y. Zu, "A highlight of recent advances in aptamer technology and its application," Molecules, vol. 20, no. 7, pp. 11959-11980, 2015.

[19] N. Nakamara, T. Matsui, Y. Ishibashi et al., "RAGE-aptamer attenuates the growth and liver metastasis of malignant melanoma in nude mice," Molecular Medicine, vol. 23, no. 1, pp. 295-306, 2017.

[20] T. Matsui, Y. Higashimoto, Y. Nishino, N. Nakamura, K. Fukami, and S. I. Yamagishi, "RAGE-aptamer blocks the development and progression of experimental diabetic nephropathy," Diabetes, vol. 66, no. 6, pp. 1683-1695, 2017.

[21] R. Li and Y.-H. Gan, "Inhibiting HDAC1 enhances the anticancer effects of statins through downregulation of GGTaseI $\beta$ expression," International Journal of Molecular Sciences, vol. 18, no. 5, p. 1010, 2017.

[22] A. Ojima, T. Matsui, S. Maeda et al., "DNA aptamer raised against advanced glycation end products inhibits melanoma growth in nude mice," Laboratory Investigation, vol. 94, no. 4, pp. 422-429, 2014.

[23] J. N. Tsoporis, S. Izhar, G. Proteau, G. Slaughter, and T. G. Parker, "S100B-RAGE dependent VEGF secretion by cardiac myocytes induces myofibroblast proliferation," Journal of Molecular and Cellular Cardiology, vol. 52, no. 2, pp. 464473, 2012.

[24] J. Li, P. W. Wu, Y. Zhou et al., "RAGE induces hepatocellular carcinoma proliferation and sorafenib resistance by modulating autophagy," Cell Death \& Disease, vol. 9, no. 2, p. 225, 2018.

[25] X. Chen, L. Zhang, I. Y. Zhang et al., "RAGE expression in tumor-associated macrophages promotes angiogenesis in glioma," Cancer Research, vol. 74, no. 24, pp. 7285-7297, 2014.

[26] C. Adamopoulos, C. Piperi, A. N. Gargalionis et al., "Advanced glycation end products upregulate lysyl oxidase and endothelin-1 in human aortic endothelial cells via parallel activation of ERK1/2-NF- $\kappa$ B and JNK-AP-1 signaling pathways," Cellular and Molecular Life Sciences, vol. 73, no. 8, pp. 16851698, 2016.

[27] A. R. Bresnick, D. J. Weber, and D. B. Zimmer, "S100 proteins in cancer," Nature Reviews. Cancer, vol. 15, no. 2, pp. 96-109, 2015.

[28] L. Seguella, F. Rinaldi, C. Marianecci et al., "Pentamidine niosomes thwart S100B effects in human colon carcinoma biopsies favouring wtp53 rescue," Journal of Cellular and Molecular Medicine, vol. 24, no. 5, pp. 3053-3063, 2020. 